\section{DOPPELGÄNGER: A FORTRAN program for simulating time-sampled spatial locations of "phantom" animals performing discrete pseudorandom walks}

\author{
DANIEL CUBICCIOTTI III \\ University of California, Davis, California 95616
}

DOPPELGÄNGER is an analy tic tool for determining whether use of space by animals that restrict their movements primarily to two dimensions (little vertical movement) differs appreciably from what would be expected if they oriented at random. The program generates Cartesian planar coordinates (X-Y pairs) simulating use of space by phantom counterparts ("doppelgängers") of individual real animals. Each animal is presumed to have been mapped on one or more "trials" (observation periods), consisting of multiple sampling occasions.

The purpose of the simulation is to provide the researcher with a set of map location data representing the movements of imaginary randomly orienting animals who start from the same locations, have the same locomotor characteristics, and are confined, if at all, by the same physical boundaries as the real animals. Thus, any difference between the spatial data of the real animal and its phantom counterpart is attributable to factors other than the restraints imposed by the physical layout of the situation or the length and duration of the locomotor "bouts" of the real animal. The coordinates produced by the program are strictly isomorphic with the real location data.

With the simulation data in hand, the researcher may compute various summary statistics and/or indexes, just as the real data are treated, and may then compare the two sets of information, providing a relatively secure inferential position from which to answer such questions as: (1) Do the real animals show significant vicinism (restriction to a smaller than expected number of quadrats)? (2) Are certain regions of the arena occupied more or less frequently than would be expected on the basis of random orientation? (3) Do particular pairs or groups of animals tend to remain closer to or further from each other than would be expected if they oriented independently? Thus, the program can assist the investigator in distinguishing orderly "patterns," "rules," or "mechanisms" that contribute to the movements of real animals from expectations based on a general null hypothesis of random navigation. In general, the pro.

This report was written in essentially finished form by D. Cubicciotti III just prior to his death. The final version was prepared by W. A. Mason. The assistance of J.P. Capitanio, W. O. Johnson, S. P. Mendoza, D. R. Rasmussen, and R.W. Summers is gratefully acknowledged. Support was provided by Grants R00169 from the National Institutes of Health and BNS 78-25384 from the National Science Foundation. gram should be most useful in the context of nonexperimental or semiexperimental inquiries about the use of space by animals residing in large and/or irregular arenas, in which solutions to the problem of how ran. domly orienting counterparts of the real animals would use the space are not immediately obvious or readily calculable.

The program generates a unique set of time-sampled map location records (Cartesian coordinates) for a phantom counterpart of each real animal. It is assumed that the real data consist of repeated "instantaneous" samples on the location of each animal, obtained at some fixed sampling interval (e.g., $15 \mathrm{~min}$ ). The set of observations on a given animal may include as many as 60 samples per "trial" (which might be a day, or a session, or some other meaningful time period) and as many as 20 trials. A maximum of $\mathbf{3 0}$ animals may be simulated in the same operation. If the researcher's interest is in interanimal distances, then all animals are assumed to have been mapped as nearly as possible at the same "instant" on each sampling occasion (Altmann, 1974).

Input. Data required to define a simulation include (1) $\mathrm{X}$ and $\mathrm{Y}$ coordinates describing $1-50$ rectangles that together define the boundaries of the arena (if any), (2) starting locations of the real animals, (3) duration of the sampling interval, (4) mean duration of a "bout" or "event" of locomotion, (5) mean speed of locomotion, and (6) scores representing the real or estimated total duration (minutes) of locomotion for each animal on each trial, or, nearly as good, scores for each animal on each trial representing "instantaneous frequency" of locomotion (Altmann, 1974), as determined by noting on each sampling occasion whether the animal was stationary or moving.

Output. Output consists of printed and punched arrays of Cartesian coordinates and printed records of the most important intermediate computations, which include net displacements achieved by each "doppelgänger" during each sampling interval and samples of the computations resulting in these displacements. The format of punched output is designed so that the data may be submitted to existing programs such as those developed by Summers (1980). These programs, which consist of a series of FORTRAN IV computer programs based on the Biomedical Computer Program P-series, compute a variety of summary measures and statistics for such time-sampled location data.

Language. DOPPELGÄNGER is written in FORTRAN IV. All computations are performed in singleprecision arithmetic on a Burroughs 6700 with a 48-bit word size; a run of intermediate size is approximately $45 \mathrm{~K}$ words. An experienced programmer can easily modify the arrays to fit a laboratory minicomputer or most microcomputers in a few hours time. No special 
functions or library routines are necessary for operation of the program.

Restrictions. Assumptions of the simulation are as follows: (1) Movements of the real animals in the vertical (up-and-down) dimension represent a negligible fraction of total travel paths; (2) the duration of a "bout" or "event" of locomotion and the speed of locomotion are both normally distributed variables, and the mean duration of a bout is briefer than the duration of the sampling interval; and (3) the parameters of locomotion (proportion of time spent locomoting, mean bout duration, mean speed), the size of the arena, and the duration of the sampling interval have values such that it is unlikely that the animal will encounter a perimeter boundary during any given sampling interval. Virtually any problem can be made to satisfy the last two assumptions by selecting an appropriate sampling interval. Any interval duration that satisfies the following inequality will meet the assumptions: $\left[(.5 \times \mathrm{S})^{2} \times(\mathrm{B})\right] /[\mathrm{V} \times \mathrm{B})^{2}$ $X \mathrm{~L} / \mathrm{T}]=$ Interval Duration $B$, where " $S$ " is the narrow est overall span of the arena, " $B$ " is the mean bout duration, " $\mathrm{V}$ " is the mean speed of locomotion, and " $\mathrm{L}$ " and " $T$ " are the mean duration of locomotion per trial and the trial duration, respectively. Sampling interval durations near the center of the acceptable range are preferable to durations near either extreme.

Computations involved in the simulation are relatively simple. The total number of locomotor bouts performed by an animal during a given trial is first estimated by dividing total duration of locomotion by mean bout duration. Individual bouts are then distributed at random among sampling intervals, and the expected net displacement achieved by the "doppelgänger" during each interval is computed from the discrete random walk formula: net distance $=\sqrt{\mathrm{N}} \times(\mathrm{L})$, where " $\mathrm{N}$ " is the number of bouts performed during the interval and " $\mathrm{L}$ " is the mean distance accomplished during an individual "step" (mean bout duration $X$ mean speed). (See Barber \& Ninham, 1970.) Each new pair of coordinates is computed on the basis of (1) the animal's most recent location, beginning with its starting location, (2) the net displacement accomplished during the interval, and (3) a randomly selected heading direction. If the problem involves a bounded arena and the computed coordinates resulting from a particular displacement and heading lie out of bounds, then a new heading is selected and computations are repeated until an "inbounds" location is achieved.

Availability. A detailed description of problem specifications and data entry procedures and a program listing can be obtained free of charge by writing W. A. Mason, California Primate Research Center, University of California, Davis, California 95616.

\section{REFERENCES}

Altmann, J. Observational study of behavior: Sampling methods. Behaviour, 1974, 49, 227-267.

Barber, M. N., \& NinhaM, B. W. Random and restricted walks: Theory and applications. New York: Gordon \& Breach Science Publishers, 1970.

Summers, R. W. Spatial ecology of a two species system of New World primates, Saimiri and Saguinus. Unpublished doctoral dissertation, University of California, Davis, 1980.

(Accepted for publication July 28, 1982.) 